Research Article

\title{
Contribution Succinonitrile additive for Performa LiTFSi Solid Polymer Electrolytes for Li-Ion Battery
}

\author{
Qolby Sabrina $^{1}{ }^{*}$, Titik Lestariningsih ${ }^{1}$, Christin Rina Ratri ${ }^{1}$, Achmad Subhan $^{1}$ \\ 1 Research Centre for Physics, Indonesian Institute of Sciences, Kompleks Puspiptek Serpong Gd. 442 \\ Tangerang Selatan, Banten 15314, Indonesia \\ * Corresponding author: qolby89@gmail.com
}

Received: 10 February 2020; Accepted: 27 May 2020; Published: 1 June 2020

\begin{abstract}
The purpose of this study is to evaluate the performance of Solid Polymer Electrolyte (SPE) which has the role of replacing liquid electrolyte in the battery system to reduce leakage. Evaluation of electrochemical SPE consisted of mixture lithium salt, solid plasticizer, and polymer precursor with different ratio. Impedance spectroscopy was used to investigate ionic conduction and dielectric response lithium bis(trifluoromethane)sulfony imide (LiTFSI) salt, and additive succinonitrile (SCN) plasticizer. The result showing enhanced high ionic conductivity. In half-cell configurations, wide electrochemical stability window of the SPE has been tested. The great potential of SPE for applications in lithium-ion batteries can be seen from the ability of window stability at room temperature. Additive $\mathrm{SCN}$ contributes to forming pores that make it easier for the Li-ion to move from the anode to the cathode and vice versa for better perform SPE. Pore of SPE has been characterization with FE-SEM. Additive $50 \%$ w.t SCN shows the best ionic conductivity with 4.2 volt wide stability window and pretty much invisible pores.
\end{abstract}

Keywords: Solid polymer electrolyte, ionic conductivity, succinonitrile, electrochemical, lithium-ion battery

\section{Introduction}

Electrolytes serve as a medium for carrying lithium ions from the anode to the cathode and preferably, therefore electrolytes must have good ionic conductivity[1]. Electrolyte solutions used in commercial lithium batteries are made from electrolyte salts dissolved in organic solvents such as Lithium hexafluorophosphate (LiPF6) salt dissolved in organic [2]. The weakness of liquid electrolytes is the potential for leakage of flammable fluids if exposed to free air and its toxic nature so it is not environmentally friendly. Another disadvantage is that it is corrosive to the electrode so that it can slowly damage overall battery performance and reduce battery life[3]. As an alternative to liquid electrolytes, electrolyte membranes are developed which do not involve organic solvents. Membrane electrolytes use electrolyte salts as ion carrying phases which are dispersed in a polymer matrix[4]. As the end result is a polymer membrane with conductive ionic properties that deliver lithium ions from the anode to the cathode and vice versa in the charging and discharging process. lithium batteries that apply electrolyte polymer membranes as ionic conductors have more guaranteed safety and structures that can be made thinner and more flexible [5].

LiTFSI (Lithium bis-trifluoromethanesulfonimide) is a hydrophilic salt that is commonly used as a source of Li-Ion in electrolytes for lithium ion batteries (LIB) as one of the better alternatives to lithium hexafluorophosphate commonly used [6], because it can increase chemical stability and thermals for electrolytes [7]. LiTFSI in EC / DMC is more ionic than LiPF6 in the same binary solvent and shows that LiTFSI in EC / DMC is a promising electrolyte salt for hybrid supercapacitors [8]. Succinonitrile (SCN) has been used as a functional additive to improve electrochemical performance of $1 \mathrm{M} \mathrm{LiPF} 6$ / EC / DEC (1: 1, volume) for high voltage LIB (cathode: $\mathrm{Li}_{1.2} \mathrm{Ni}_{0.2} \mathrm{Mn}_{0.6} \mathrm{O}_{2}$, anode : $\mathrm{Li}$ ) [9]. Therefore, it is imperative to develop new SPE with additive SCN to get large pore size, high porosity and high ionic conductivity for secondary Li-ion battery application on the high voltage. 


\section{Materials and Methods}

\section{Materials}

Material electrolyte salt lithium bis(trifluoromethane)sulfony imide (LiTFSI) from Sigma Aldrich. 0.5 M LiTFSi salt was dissolved in the EC: EMC (3:7) purchased from Merck with purity $>98 \%$ as the activator it,s considered. Pellets Poly(vinylidene fluoride-co-hexafluoropropylene) (PVdF HFP, Sigma Aldrich) with a molecular weight $>180$ andvpurity $>98 \%$ as polymer matrixes. N,N-dimethylacetamide (DMAC purity $>99 \%$, Merck) wich its considered as solvent.

\section{Methods}

Synthesis

In preparing the SPE, firstly dispersion of SCN in the salt solution, next polymer materials were dissolved in DMAC using magnetic stirrer on a hot plate $60^{\circ} \mathrm{C}, 60 \mathrm{rpm}$ for 10 hours. To fabricate a solvent-casting type plate by the solvent-casting method.

\section{Characterization}

Electrochemical Impedance Spectroscopy. Electrochemical Impedance Spectroscopy (EIS) is a powerful tool to study the complex electrochemical processes at electrodes and interface of solid polymer electrolyte sample. EIS EIS (Electrochemical Impedance Spectroscopy) to determine the value of impedance or resistance of a sample, this impedance value will be used to calculate the amount of ion conductivity that a sheet has based on its surface area, thickness and particle distribution. The EIS analysis can qualitatively determine the information such as electronic/ionic conduction in electrode/electrolyte, charge transfer kinetics at the interface, the interfacial charging effect at the surface films or the interfacial double layer. The EIS was widely applied on electrochemistry studies of lithium-ion batteries. To characterize the ionic conductivity and the interfacial resistance of electrolyte by EIS, first, a testing cell for the ionic conductivity measurement was designed and assembled. The analysis of impedance spectra is performed in terms of Nyquist plots where the imaginary part of the impedance is plotted as a function of the real part over a wide range of frequencies, for example, a frequency from $0.1 \mathrm{~Hz}-1 \mathrm{MHz}$ at the AC voltage of $10 \mathrm{mV}$.

Linear sweep voltammetry (LSV) is a voltammetry method where the current at the working electrode is measured while the potential between the reference work electrode is swept linearly in time [10]. In LSV, scanning is performed from a lower potential limit to a higher one. The characteristics of LSV depend on the rate of electron transfer reactions, chemical reactivity of electroactive species and potential scanning rates [11].

Field Emission Scanning Electron Microscopy. The morphological features of the prepared membranes were examined using the Field Emission Scanning Electron Microscopy JEOL. low acceleration voltage of $3 \mathrm{kV}$ and probe current 6 was used, to preserve sharpness, focus and to avoid sample damage.

\section{Results and Discussion}

The addition of succinonitrile can increase the ion conductivity, where SCN will decrease the crystallinity of the polymer matrix so that it is more plastic and helps to separate lithium ions from their salts, increase ion mobility, and produce high ionic conductivity [12]. The addition of SCN to the electrolyte shows high ambient conductivity, provided that the mixing process occurs homogeneously. at the addition of $40 \% \mathrm{SCN}$ it appears ion mobility is inhibited so that the ionic conductivity value is low, this is explained in more detail in the SPE morphology. High ionic conductivity in 50\% SCN will facilitate the electrochemical process in batteries, especially in the intercalation process of Lithium-ion distribution [13]. Table 1 describes value ionic conductivity of Solid Polymer Electrolyte. 
Table 1. The ionic conductivity of Solid Polymer Electrolyte

\begin{tabular}{ccc}
\hline Sample & SCN(Additive) & Ionic conductivity $(\sigma)\left({\left.\mathrm{S} . \mathrm{cm}^{-1}\right)}^{-1}\right.$ \\
$\mathrm{a}$ & $0 \%$ & $4,74 \times 10^{-4}$ \\
$\mathrm{~b}$ & $40 \%$ & $7,88 \times 10^{-5}$ \\
$\mathrm{c}$ & $50 \%$ & $5,47 \times 10^{-4}$ \\
\hline
\end{tabular}
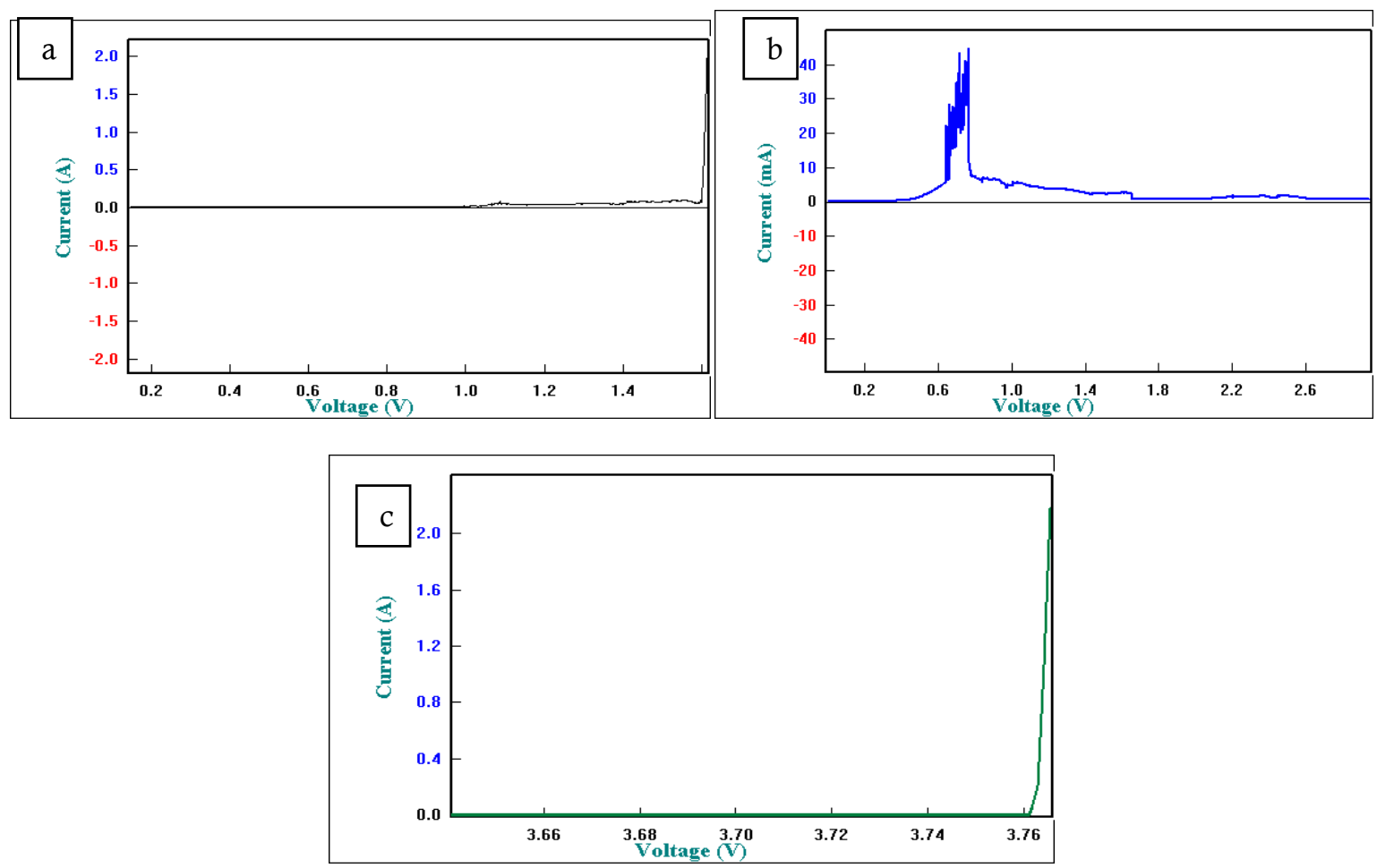

Figure 1. Linear sweep voltammetry (LSV) (a) current changes with variation in voltage SCN0\%, (b) SCN40\%, (c) $\mathrm{SCN} 50 \%$. 
Figure 1. shows that succinonitrile $(\mathrm{SCN})$ as a functional additive to improve thermal stability and expand the electrochemical window of electrolyte oxidation. inhibition of ion mobility at $40 \% \mathrm{SCN}$ is shown by the limited stability of the window voltage. The stability of $50 \%$ SCN showing voltage window bigger because of increased ion mobility.
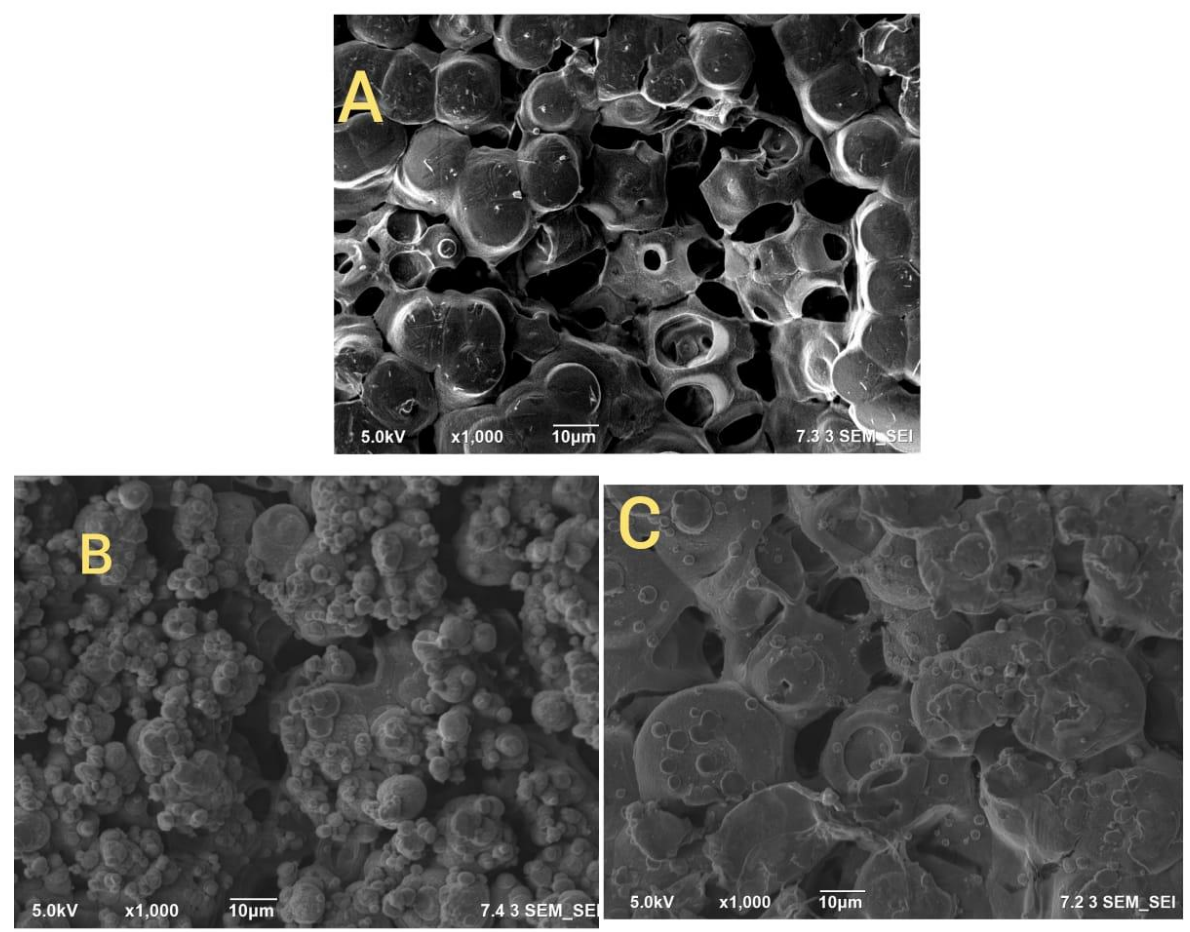

Figure 2. Morphology solid electrolyte (a) SCN0\%, (b) SCN40\%, (c) SCN50\%.

The size of the particles looks the same, round with the same size. The width of the gap is varied, there are large porous and small porous. The difference in the number and pore size of the three different solid polymer electrolytes is due to the influence of SCN which SCN can open pores in the SPE so that the distribution of ions for charge and discharge is better. Composition without SCN with FESEM test can be seen in Figure 2. Based on Figure 2. (A), it can be seen that the results of the SPE deposition are homogeneous. The size of the particles looks the same, round with the same size, the average diameter of the pore size was $13.484 \mu \mathrm{m}$. Measurement using image. Figure 2.(B) SCN $40 \%$ by FESEM testing where the particle size is not the same, the presence of agglomeration can be distinctly observed in the case of sample synthesized and not homogeneous deposition. There are small and large particles, the width of the gap is wider and elongated. Average diameter of the pore size was $11,027 \mu \mathrm{m}$. Fig.2.(C) SCN $50 \%$ by FESEM testing obtained homogeneous deposited particles with larger pore sizes. Average diameter of the pore size was 5,502 $\mu \mathrm{m}$. Material electrolyte composition and morphology SPE can bring about a huge jump in performance such as poor microstructure with small porosity have low stability performance [14]. Matrix pore size plays an important role in the conductivity enhancement and agglomeration can also affect the distribution of the lithium salt and matrix particles in the SPE[15].

\section{Conclusion}

Solid electrolyte based LiTFSi with fluorinated polymers (PVDF/PVDF HFP) with SCN additive were successfully prepared. However, Solid electrolyte consist of PVDF HFP and LiTFSi with SCN additive produces a perforated sheet. The ionic conductivity of cell employed solid electrolyte without additive SCN and with additive SCN respectively increase from $4.74 \times 10^{-4}$ to $5.47 \times 10^{-4}$. FESEM testing obtained homogeneous deposited particles with larger pore sizes. The average diameter of the pore size was decreased from $13.484 \mu \mathrm{m}$ to $5.502 \mu \mathrm{m}$ with the number of SCN additives. The stability of the resulting voltage window widened as the number of $\mathrm{SCN}$ additives. 


\section{Acknowledgement}

This work was supported by Research Centre for Physics Department- Indonesian Institute of Sciences and Ministry of Research and Technology and Higher Education Indonesia through Insentif Riset Sistem Inovasi Nasional (INSINAS) grant Lithium extraction from Indonesian raw materials for lithium battery budget year 2019 .

\section{References}

[1] S. Sadhukhan, Preparation and Characterization of Polymer Electrolytes (2011).

[2] Q. Lia, J. Chenb, L. Fan, X. Kong, Y. Lu, Progress in electrolytes for rechargeable Li-based batteries and beyond, Green Energy \& Environment (2016).

[3] R.J.Brodd, Batteries for sustainability: selected entries from the encyclopedia of sustainability science and technology, Springer Science \& Business Media (2012).

[4] P.J. Alarco, Y. Abu-Lebdeh, Abouimrane A, Armand M, The plastic-crystalline phase of succinonitrile as a universal matrix for solid-state ionic conductors. Nature materials 3: 476 (2004).

[5] X. Chen, W. Xu, M.H. Engelhard, J. Zheng, Y. Zhang, et al, Mixed salts of LiTFSI and LiBOB for stable LiFePO 4-based batteries at elevated temperatures, Journal of Materials Chemistry $A$ 2: 234652 (2014).

[6] L.Z. Fan, Y.S. Hu, A.J. Bhattacharyya, J. Maier, Succinonitrile as a versatile additive for Polymer Electrolytes (2011).

[7] S.A. Freunberger, Y. Chen, Z. Peng, M.J. Griffin, L.J. Hardwick. et al, Reactions in the Rechargeable Lithium-O2 Battery with Alkyl Carbonate Electrolytes. Journal of the American Chemical Society 133: 8040-47 (2011).

[8] M. Hietaniemi, Thermal stability of chemicals used in lithium-ion batteries. Master thesis,(2015)

[9] T. Liu, Z. Chang, Y. Yin, K. Chen, Y. Zhang, X. Zhang, The PVDF-HFP gel polymer electrolyte for Li-O2 battery, Solid State Ionics 318: 88-94 (2018).

[10] K. Matsumoto, K. Inoue, K. Nakahara, R. Yuge, T. Noguchi, K. Utsugi, Suppression of aluminum corrosion by using high concentration LiTFSI electrolyte, Journal of Power Sources 231: 234-38, (2013).

[11] M. Noor, M. Careem, Majid, A. Arof, Characterisation of plasticised PVDF-HFP polymer electrolytes. Materials Research Innovations 15: s157-s60 (2011).

[12] T. Reddy, Linden's handbook of batteries. New York, NY: McGraw-Hill: 1200 (2011).

[13] V.K. Singh, R.K. Singh, Development of ion conducting polymer gel electrolyte membranes based on polymer PVdF-HFP, BMIMTFSI ionic liquid and the Li-salt with improved electrical, thermal and structural properties. Journal of Materials Chemistry C 3: 7305-18, (2015).

[14] S. Li, Y. M. Chen, W. Liang, Y. Shao, K. Liu, Z. Nikolov, Y. Zhu, A Superionic Conductive Electrochemically Stable Dual-Salt Polymer Electrolyte. Joule, (2018).

[15] X.Chen, P.M. Vereecken, Solid and Solid-Like Composite Electrolyte for Lithium Ion Batteries: Engineering the Ion Conductivity at Interfaces. Adv. Mater. Interfaces, (2018). 